\title{
Stochastic Patrolling and Collision Avoidance for Two UAVs in a Base Defense Scenario
}

\author{
Justin Jackson, ${ }^{*}$ \\ University of Michigan, Ann Arbor, Michigan, 48109, United States \\ Ricardo Bencatel ${ }^{\dagger}$ \\ Universidade do Porto, 4200-465, Porto, Portugal \\ Zahid Hasan $\ddagger$ \\ University of Michigan, Ann Arbor, Michigan, 48109, United States \\ and Anouck Girard ${ }^{\S}$ \\ University of Michigan, Ann Arbor, Michigan, 48109, United States
}

\begin{abstract}
This paper discusses the stochastic patrolling of a region under collision avoidance constraints by two UAVs. The patrol pattern is controlled by an algorithm that ensures weighted randomized coverage of the region in such a way as to patrol the entire region. The purpose is to search a region for an intruder by a pattern that is undetectable to the intruder. The algorithm ensures that the entire region is searched in an unpredictable way. Avoidance algorithms are implemented to adjust the trajectories of the UAVs in a relatively confined space. The control structure presented is hierarchical with operator-inthe-loop capability as well as lower level collision avoidance control to ensure safe guidance. Computational results are presented detailing the implementation of these algorithms on autonomous helicopters.
\end{abstract}

Table 1. Nomenclature

\section{Symbol Description \\ $r_{1} \quad$ Inertial position of UAV 1 \\ $r_{2} \quad$ Inertial position of UAV 2 \\ $\psi_{1} \quad$ Heading angle of UAV 1 \\ $\psi_{2} \quad$ Heading angle of UAV 2 \\ $r_{w p_{1}} \quad$ Inertial position of waypoint for UAV 1 \\ $r_{w p_{2}} \quad$ Inertial position of waypoint for UAV 2 \\ $r_{t p_{1}} \quad$ Inertial position of target point for UAV 1 \\ $r_{t p_{2}} \quad$ Inertial position of target point for UAV 2 \\ $r_{12} \quad$ Vector from UAV 1 to UAV 2 \\ $r_{a} \quad \mathrm{UAV}$ avoidance radius \\ $r_{s} \quad$ UAV safe radius

\footnotetext{
$\dagger$ Graduate Student

$\ddagger$ Undergraduate Student, Aerospace Engineering

$\S$ Assistant Professor, Aerospace Engineering
} \\ * Graduate Student, Aerospace Engineering, Student Member.}

$R_{1} \quad$ Direction cosine matrix transforms vectors from inertial axes to UAV 1 heading frame

$R_{2} \quad$ Direction cosine matrix transforms vectors from inertial axes to UAV 2 heading frame

$\phi_{F L} \quad$ Angle of vector pointing from the leader UAV to the follower UAV relative to inertial x-axis 


\section{Introduction}

This paper describes the development and implementation of an algorithm that is used to navigate two unmanned aerial vehicles (UAVs). The UAVs stochastically patrol a region and use a method of collision avoidance that adjusts the flight path of the UAVs to navigate them in a de-conflicted manner. The stochastic search algorithm is a policy that selects waypoints in this region. The patrol region is divided into individual search areas. The centers of these regions are the waypoints that are selected by the search algorithm for visitation. The weighted probability of selecting a waypoint is set to zero when it is selected and increases as other waypoints in the region are visited. This effectively reduces the likelihood that a UAV will remain in a portion of the search region after visitation.

A deconfliction algorithm computes the intersection between the flight paths of the UAVs to determine if there is a danger of collision. The algorithm then selects one UAV to be the leader and the other to be the follower. The one that is farthest from the flight path intersection is the follower and yields right-of-way to the leader, Fig 3(a). The path of the leader UAV remains unchanged until it reaches its waypoint unless a collision becomes imminent or it is re-designated as follower. When a waypoint is reached, the corresponding portion of the search area is considered searched and a new waypoint is given by the stochastic patrol algorithm.

\section{A. Motivation}

The central problem that this work addresses is the need to have two UAVs patrol an area in a manner that is unpredictable to an outside observer, but that insures the coverage of the entire area as the system evolves in time. This ability is applicable to border patrol, ${ }^{1}$ maritime patrol ${ }^{2}$ and base defense scenarios. ${ }^{2}$ The guidance strategy presented in this paper is designed for two miniature helicopters patrolling a mock base in close proximity to each other as part of an indoor testbed for high level control algorithms. The patrolling algorithm must be able to patrol a region that is partitioned into a grid. The UAVs must also navigate in an effective and de-conflicted manner. The UAVs fly at the same altitude, hence, only 2D navigation is considered. The requirement for the algorithm to run in real time is central to this work.

\section{B. Literature review}

Aircraft collision avoidance is a current and challenging control topic. Bicchi and Pallottino presented a tutorial at ICRA 2006 with an extensive overview of collision avoidance methods. ${ }^{3}$ Collision avoidance for groups of two or more is of particular interest. Formation control deals with organization of UAVs into useful configurations and maintaining the stability of these formations in the presence of disturbances. ${ }^{4}$ The Traffic Alert and Collision Avoidance System (TCAS) was created to avoid aircraft collisions, acting only as a last resort system. ${ }^{5}$ Other research is concerned with collision free navigation of large numbers of UAVs within a confined area. ${ }^{6}$

Researchers have used various optimal control frameworks to gain a valuable insight on the problem structure and possible solutions. ${ }^{7-11}$ The biggest handicap on these methods is the centralized formulation and its scalability. Jardin presents an optimal control method to guide two aircraft and avoid collisions between them. ${ }^{12}$ This method can maintain near optimal paths in the presence of collision avoidance maneuvers. Borrelli et al. ${ }^{13}$ describe distributed Receding Horizon Control (RHC) as a feasible solution for a large number of aircraft. Often problems of waypoint seeking and collision avoidance can be solved as constrained optimization problems, using a linear approximation to constraints to quickly solve the problem of navigating UAVs to avoid each other and stationary obstacles. ${ }^{14}$ Groups of UAVs can be navigated to waypoints without collision through the use artificial potential fields. ${ }^{15}$ The use of artificial potentials is attractive because the methods are computationally tractable and result in global navigation of an entire group, however, care must be taken to ensure that the UAVs do not become trapped where the sum of potential forces is zero. Tomlin et al. have presented a method to avoid collisions in free flight using a hybrid control system model to study and generate collision avoidance maneuvers for two aircraft in a decentralized manner. ${ }^{16}$ Hoffmann and Tomlin developed and demonstrated decentralized collision avoidance algorithms for Vertical Takeoff and Landing (VTOL) vehicles. ${ }^{17}$ They present one algorithm for two vehicles and a second algorithm for several vehicles. These algorithms have been shown to be safe and to be of low computational cost. Experiments were performed with STARMAC. ${ }^{18}$ 
Another problem addressed in the literature is the allocation of teams of UAVs to accomplish various tasks. A subset of this issue is the patrolling of a border or region of interest by more than one UAV. Optimization problems can be posed such that multiple vehicles patrol a region and track multiple targets. ${ }^{19}$ The problem of border patrol by multiple UAVs deals with how to allocate UAVs while maintaining the ability to track moving targets. ${ }^{1}$ Pallottino et al. address the problem of complex hybrid automata verification for decentralized control systems. ${ }^{20}$ The authors use probabilistic verification, as classical approaches are difficult to apply.

\section{Original contributions}

An algorithm is presented here that successfully navigates two UAVs in a confined space. A hierarchical approach is presented that allows an operator to specify a search region without being concerned with the details of flight path de-confliction. This region is patrolled stochastically so that an outside observer is unable to discern a predictable pattern. The path planning algorithm runs in real time and is effective in navigating the UAVs in a collision free manner. This work presents the development of the stochastic patrolling method and the collision avoidance protocols. Simulation results demonstrate the viability of this method to navigate the UAVs.

\section{Overview}

This paper is organized as such. Section II discusses the formulation of the problem and the method used for patrolling the search region in a minimally predictable and effective manner. Section III describes the results of the algorithms used for stochastic patrolling, collision avoidance and deconfliction. Section IV presents conclusions drawn from this work and section $\mathrm{V}$ contains a discussion of future work.

\section{Problem formulation}

The UAVs patrol within a finite region at a constant altitude. The region that the UAVs search need not be a uniform grid, all that is required is a list of waypoints that are to be visited. These waypoints represent the centers of the various subregions that the search region is divided into. These waypoints are repeatedly visited to search for intruders. The UAVs are guided by the layered control architecture that is illustrated in Fig. 1

\section{A. Assumptions and modeling}

An aircraft standard, north-east-down coordinate system is used. The UAVs are modeled using a constant velocity assumption. It is assumed that they can instantaneously change heading. The unicycle model is used for the vehicle kinematics.

$$
\begin{aligned}
\dot{x_{i}} & =V \cos \left(\psi_{i}\right) \\
\dot{y_{i}} & =V \sin \left(\psi_{i}\right) \\
\psi & =u_{i}
\end{aligned}
$$

In the patrolling scenario, a sensor is used to detect the intruder. The UAVs are assumed to fly at the optimal altitude for the sensor used to detect intruders. The avoidance algorithm assumes constant altitude. Collision avoidance and de-confliction are performed for a pair of vehicles.

\section{B. Stochastic patrolling}

The region patrolled by the team of UAVs is divided into discrete waypoints to be visited. These points need not lie on a uniform grid. The patrolling algorithm searches all points repeatedly in a minimally predictable way as viewed from an intruder. To accomplish this, the sectors are visited in a pattern dictated by a weighted random selection. The initial weightings of the sectors are defined by the operator. The sum over all of the sector weightings must be equal to one. In the authors' examples each sector initially has equal weighting and equal chance of being selected. 


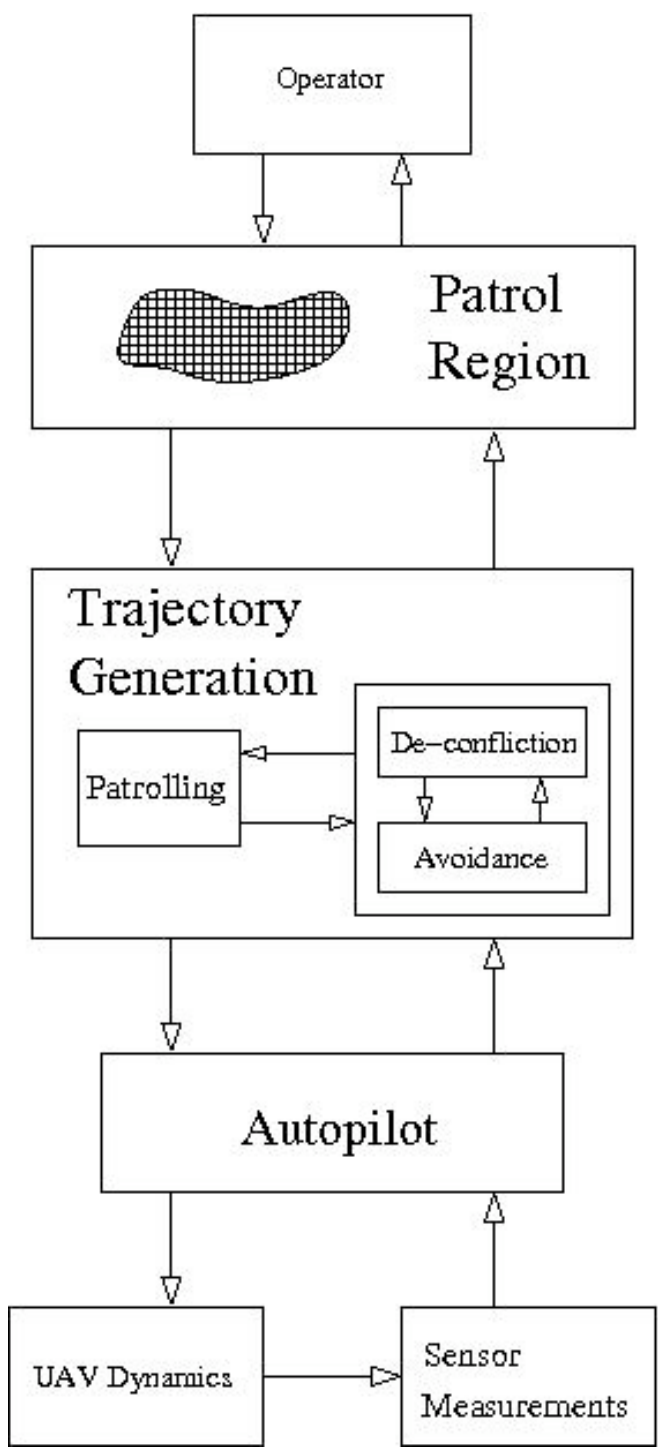

Figure 1. Illustration of high level control architecture with operator in the loop. 
A search area is selected by generating a uniform random number and weighting the selection according to the search weighting. After a point is selected, the weighting for that sector goes to zero. The weighting that the selected sector had before selection, $W_{i_{k}}^{s}$, is then distributed among the remaining sectors to maintain the sum of the weightings equal to one. Initially, the search weighting for each sector is

$$
W_{i}=\frac{1}{N},
$$

where $N$ is the number of sectors that the search region is divided into. After the weighted random selection of a sector for visitation, the search weighting of each unselected sector becomes,

$$
W_{i_{k+1}}^{u}=W_{i_{k}}^{u}+\frac{1}{N-1} W_{i_{k}}^{s}
$$

where $W_{i_{k}}^{s}$ is the weighting of the selected sector prior to selection. When a sector is selected, its weighting becomes,

$$
W_{i_{k+1}}^{s}=0
$$

The probability of being selected for any sector in the coverage region is

$$
P_{i}=W_{i} .
$$

The selection of a sector for visitation is done by generating a random number $w$ between 0 and 1 , and successively summing the weightings of the sectors until the sum exceeds $w$. The last sector added to the sum is then selected for visitation. The initial expected value of the sector weights, $E\left(W_{i_{0}}\right)=\frac{1}{N}$ by Eq. 4 . From the initial weighting distribution,

$$
\sum_{i=1}^{N} W_{i_{0}}=1
$$

To show that $E\left(W_{i_{k}}\right)=\frac{1}{N}$, for all time, we start with,

$$
\begin{aligned}
\sum_{i=1}^{N} W_{i_{k+1}} & =\sum_{i=1}^{N-1} W_{i_{k+1}}^{u}+W_{i_{k+1}}^{s}, \\
& =\sum_{i=1}^{N-1} W_{i_{k+1}}^{u},
\end{aligned}
$$

by Eq. 6. Eq. 5 is substituted into Eq. 10 to obtain,

$$
\begin{aligned}
\sum_{i=1}^{N} W_{i_{k+1}} & =\sum_{i=1}^{N-1} W_{i_{k}}^{u}+(N-1)\left(\frac{1}{N-1} W_{k}^{s}\right) \\
& =\sum_{i=1}^{N-1} W_{i_{k}}^{u}+W_{k}^{s}
\end{aligned}
$$

From this, we see that,

$$
\begin{aligned}
\sum_{i=1}^{N} W_{i_{k+1}} & =\sum_{i=1}^{N} W_{i_{k}}, \\
& =1,
\end{aligned}
$$

by substituting the initial sum of the weightings from Eq. 8. The expectation becomes, 


$$
\begin{aligned}
E\left(W_{i_{k+1}}\right) & =\frac{\sum_{i=1}^{N} W_{i_{k+1}}}{N}, \\
& =\frac{\sum_{i=1}^{N} W_{i_{k}}}{N}, \\
& =E\left(W_{i_{k}}\right) .
\end{aligned}
$$

Thus, the expected value of a sector's search weighting does not change. Table 2 shows the distribution of the search weightings of each sector at the time $k=0$. Table 3 shows the weighting distribution at time $k=1$. The starting point of the UAVs can be anywhere in the patrolled region. The portion labeled base is excluded from visitation as it is considered to be a fortified area.

Table 2. section selection weightings, $\mathrm{k}=0$

\begin{tabular}{llll}
$\mathbf{W}$ & $\mathbf{1}$ & $\mathbf{2}$ & $\mathbf{3}$ \\
$\mathbf{1}$ & 0.1250 & 0.1250 & 0.1250 \\
$\mathbf{2}$ & 0.1250 & base & 0.1250 \\
$\mathbf{3}$ & 0.1250 & 0.1250 & 0.1250 \\
\hline
\end{tabular}

Table 3. section selection weightings, $\mathrm{k}=1$

\begin{tabular}{llll}
\hline $\mathbf{W}$ & $\mathbf{1}$ & $\mathbf{2}$ & $\mathbf{3}$ \\
$\mathbf{1}$ & 0 & 0.1429 & 0.1429 \\
$\mathbf{2}$ & 0.1429 & base & 0.1429 \\
$\mathbf{3}$ & 0.1429 & 0.1429 & 0.1429 \\
\hline
\end{tabular}

\section{Avoidance algorithm}

The two UAVs are each given waypoints within a restricted grid and must navigate without collision. The algorithm that accomplishes this also has to run in real time. This is accomplished with two levels of avoidance control. The first level is the most basic and is in place to deal with emergencies, this is the collision avoidance level. The second level employs a method of trajectory planning that guides the UAVs so that one yields right-of-way to the other. These two algorithms function to temporarily replace the waypoints given by the stochastic patrolling algorithm with an intermediate target point. Tracking this target point directs the UAVs out of harm's way.

\section{Collision avoidance}

The collision avoidance algorithm is responsible for maintaining a specified separation distance between the two UAVs. Two circular zones are created around each UAV to accomplish this. The first is a safe zone defined by the radius $r_{s}$ and the second is an avoidance zone defined by the radius $r_{a}$, Fig. 2 . These define the separation distance between the centers of the two UAVs. If

$$
\left\|r_{12}\right\| \leq r_{s}
$$

collision avoidance is required for both UAVs. The vector $r_{12}$ is the vector from UAV 1 to UAV 2 . The new heading angles of each of the UAVs are

$$
\begin{aligned}
& \psi_{1}=\arctan \frac{r_{1 y}-r_{2_{y}}}{r_{1_{x}}-r_{2_{x}}}, \\
& \psi_{2}=\arctan \frac{r_{2 y}-r_{1_{y}}}{r_{2_{x}}-r_{1_{x}}},
\end{aligned}
$$


where $r_{i x}$ and $r_{i y}$ are the components of the UAV position vectors. The new target points for this case are,

$$
\begin{gathered}
r_{t p_{1}}=r_{1}+r_{a}\left[\cos \psi_{1}, \sin \psi_{1}\right]^{T}, \\
r_{t p_{2}}=r_{2}+r_{a}\left[\cos \psi_{2}, \sin \psi_{2}\right]^{T} .
\end{gathered}
$$

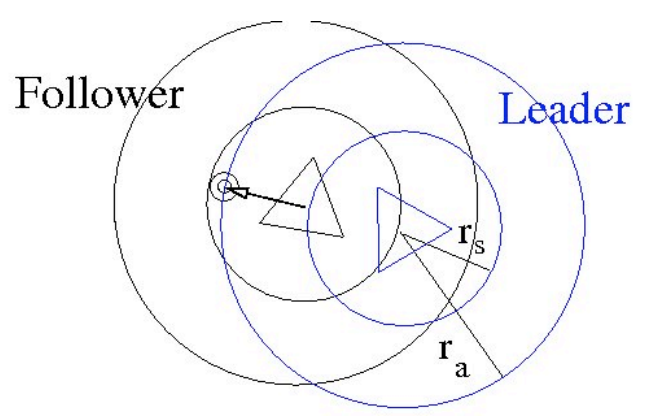

$\triangle$ Vehicle

(c) Target point

Figure 2. Emergency collision avoidance.

\section{Deconfliction algorithm}

The purpose of the deconfliction algorithm is to determine if there is a possible flight path intersection and redirect the UAVs' flight paths to avoid the intersection. The centers of gravity of the UAVs should maintain a separation distance of $r_{a}$. Every time a new waypoint is given, the intersection of the UAV paths is computed. This intersection is based on the intersection between the two lines that are drawn from each UAV's phantom position to its phantom waypoint, Fig 3(a). The phantom positions and waypoints of each UAV are defined as

$$
\begin{aligned}
& p_{1}=r_{1}+\frac{r_{a}}{\left\|r_{w p_{1}}-r_{1}\right\|}\left(r_{1}-r_{w p_{1}}\right), \\
& p_{2}=r_{2}+\frac{r_{a}}{\left\|r_{w p_{2}}-r_{2}\right\|}\left(r_{2}-r_{w p_{2}}\right),
\end{aligned}
$$

and

$$
\begin{aligned}
& p_{w p_{1}}=r_{w p_{1}}+\frac{r_{a}}{\left\|r_{w p_{1}}-r_{1}\right\|}\left(r_{w p_{1}}-r_{1}\right), \\
& p_{w p_{2}}=r_{w p_{2}}+\frac{r_{a}}{\left\|r_{w p_{2}}-r_{2}\right\|}\left(r_{w p_{2}}-r_{2}\right) .
\end{aligned}
$$

Here, $r_{w p_{i}}$ and $p_{w p_{i}}$ are the actual waypoint position and the phantom waypoint position. The intersection of the paths of the two UAVs is found from the intersection of the path of the second UAV with the first by

$$
\begin{aligned}
{\left[x_{i n t}\right]_{1} } & =\left[p_{2_{x}}\right]_{1}-\frac{\left(\left[p_{w p_{2_{x}}}\right]_{1}-\left[p_{1_{x}}\right]_{1}\right)}{\left(\left[p_{w p_{2_{y}}}\right]_{1}-\left[p_{1_{y}}\right]_{1}\right)}\left[p_{2_{y}}\right]_{1}, \\
r_{\text {int }} & =\left[R_{1}\right]\left[x_{i n t}, 0\right]_{1}^{T}
\end{aligned}
$$

where the bracket notation, $[*]_{1}$ indicates that the quantity is expressed in a frame with origin at UAV 1 and $\mathrm{x}$-axis in the direction of the waypoint of UAV 1. The leader UAV is designated to be the one for which,

$$
i=\arg \min _{j}\left\|r_{j}-r_{\text {int }}\right\|, \quad j=1,2
$$

$$
7 \text { of } 11
$$


The target point for the leader UAV remains its waypoint and its commanded heading angle remains unchanged unless emergency collision avoidance becomes necessary. The new target point for the follower UAV becomes,

$$
\begin{aligned}
r_{t p_{F}} & =r_{L}+r_{a}\left[\cos \left(\phi_{F L}+\phi_{t}\right), \sin \left(\phi_{F L}+\phi_{t}\right)\right]^{T}, \\
\phi_{t} & =\arccos \frac{r_{a}}{\left\|r_{12}\right\|} .
\end{aligned}
$$

where $\phi_{t}$ is the additional angle offset that points from the leader UAV to the tangent point. The sign of $\phi_{t}$ is adjusted to be negative or positive so as to place the target point on the opposite side of the leader UAV as its waypoint. This ensures that the follower UAV moves to the aft of the leader UAV. After the intersection has been avoided, either the follower UAV has crossed the flight path of the leader UAV or the leader UAV has passed the original intersection point, the follower UAV continues in a straight line toward its waypoint.

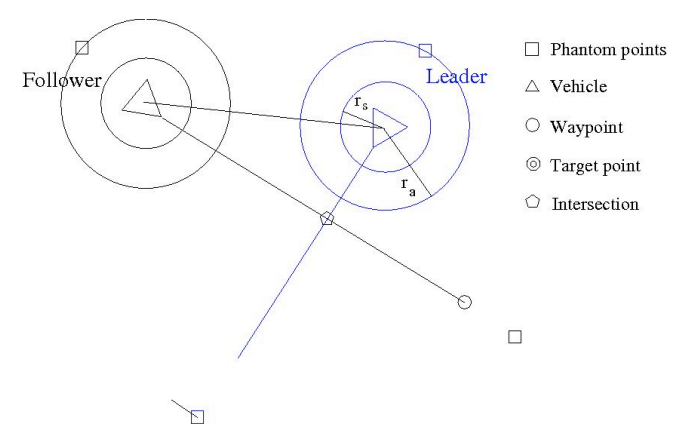

(a) Intersection point computation.

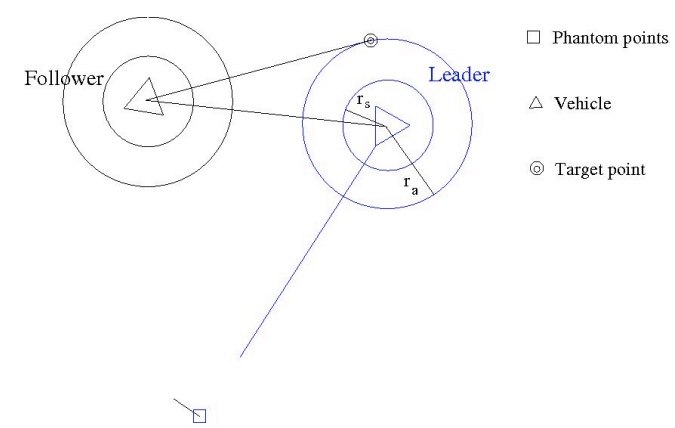

(b) Target point computation.

Figure 3. Deconfliction example.

If the follower begins with an initial position more than a distance $r_{a}$ from the constant flight path of the leader, then de-confliction will always be successful and collision avoidance will not be needed. We can see this by investigating the behavior of this guidance law when the follower reaches the avoidance boundary of the leader. If the follower is outside of the distance $r_{a}$ from the flight path of the leader UAV, its velocity

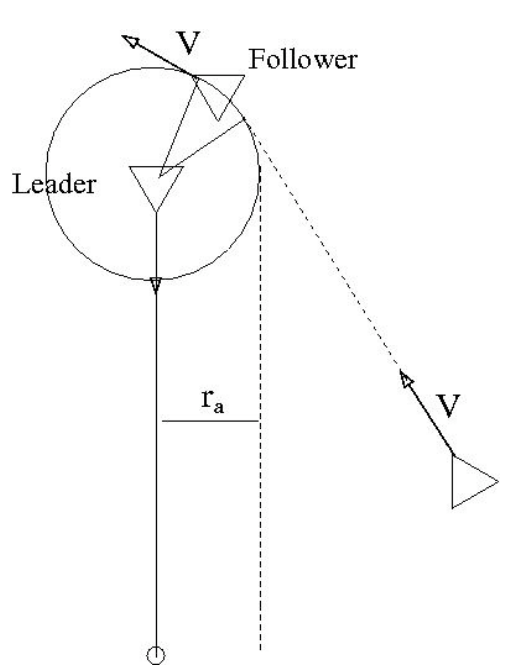

(a) Successful de-confliction - leader does not intrude into the follower's avoidance zone.

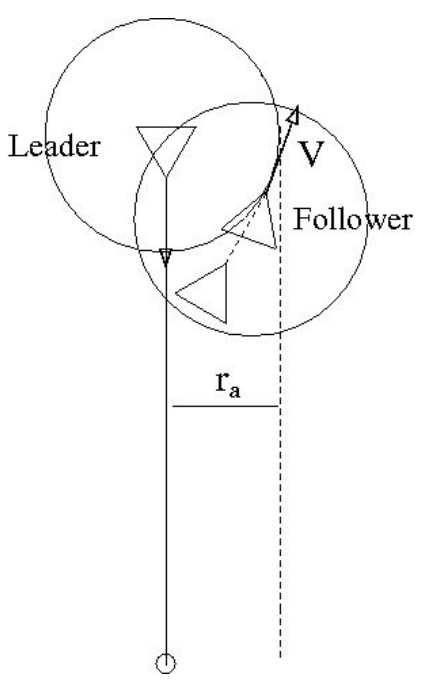

(b) Unsuccessful de-confliction - leader intrudes into the follower's avoidance zone.

Figure 4. Requirements for successful de-confliction. 
will always direct it to a point aft of the leader with velocity tangent to the leader's avoidance zone. If the follower reaches the boundary of the leader's avoidance zone, the velocity vector of the leader will point away from the follower's avoidance zone and the leader will not intrude on the follower's avoidance zone, figure $4(\mathrm{a})$.

Consider the case where the follower UAV begins with initial position within distance $r_{a}$ from the leader's flight path. The follower can reach the boundary of the leader's avoidance zone within a distance $r_{a}$ of the leader's flight path. In this case, the velocity vector of the leader points in the direction of the follower's avoidance zone. In this case, collision avoidance will be needed when the leader intrudes into the follower's avoidance zone, figure 4 (b).

\section{Results}

This section presents simulation results from the patrolling and avoidance algorithms. Results from simulation are presented that show a representative generation of waypoints and one de-confliction scenario. The UAVs successfully designate a leader and follower and the follower yields right-of-way to the leader.

\section{Simulation results}

Figure 5(a) shows the paths of the UAVs as they visit their respective waypoints. The leader UAV visits three waypoints. The follower has a conflict and must move to avoid the leader after it visits its first waypoint. Note that the follower is not selected as such until after there is a perceived conflict. Figure 5(b) depicts the separation distance between the two UAVs. If the separation drops below the lower bounding dotted line, or safe radius $r_{s}$, collision avoidance is required. The separation remains above this lower bound. The higher bound is the avoidance radius, $r_{a}$ that the UAVs track in the event of safe radius violation.

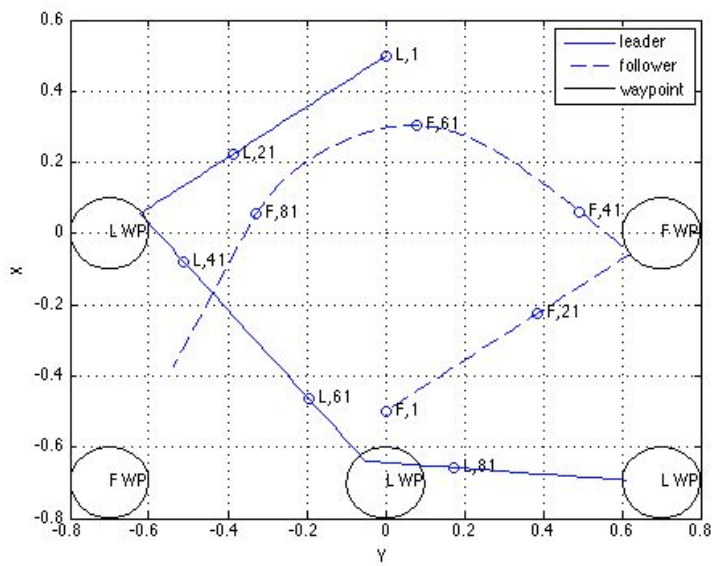

(a) Leader and follower paths.

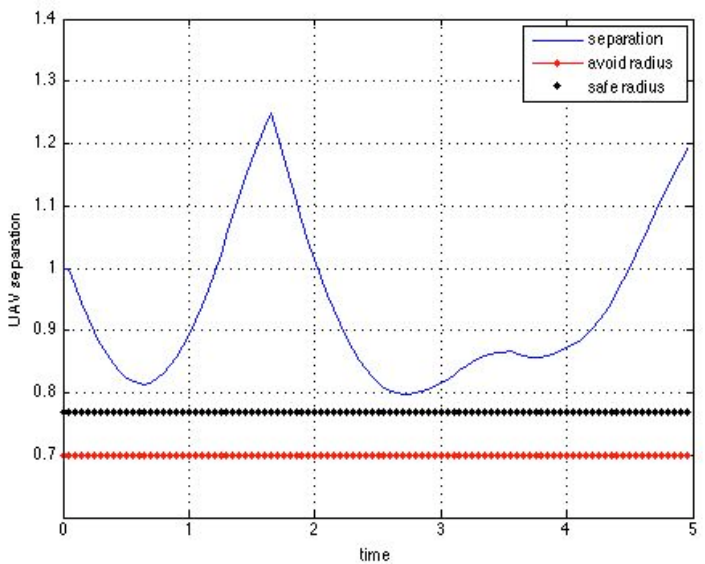

(b) UAV separation distance.

Figure 5. Simulation example with avoidance satisfaction.

Figures 6(a) and 6(b) show the distance between the leader UAV, follower UAV and their respective waypoints. For the leader, this distance drops linearly as it moves in a straight line towards its waypoint and rises again as the waypoint switches to the next point generated by the stochastic patrolling algorithm. The follower UAV visits its first waypoint similarly, but is diverted by the de-confliction algorithm while in route to its second waypoint. The collision avoidance algorithm is not used here because the UAVs maintain adequate separation distance.

\section{Conclusions}

The authors have presented a method for patrolling a region in a stochastic manner that ensures the coverage of a region. Flight path conflicts are resolved by selecting the UAV closest to the flight path intersection to have right-of-way. The UAV farthest from the flight path intersection navigates to avoid the 


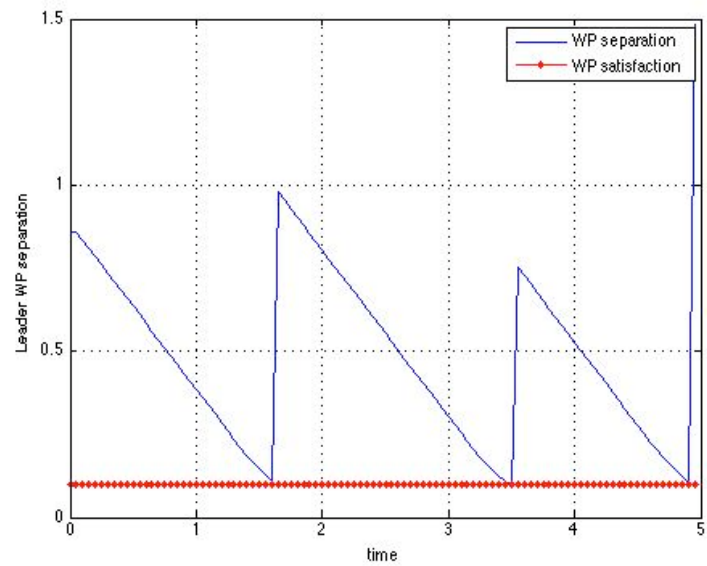

(a) Leader distance from waypoint.

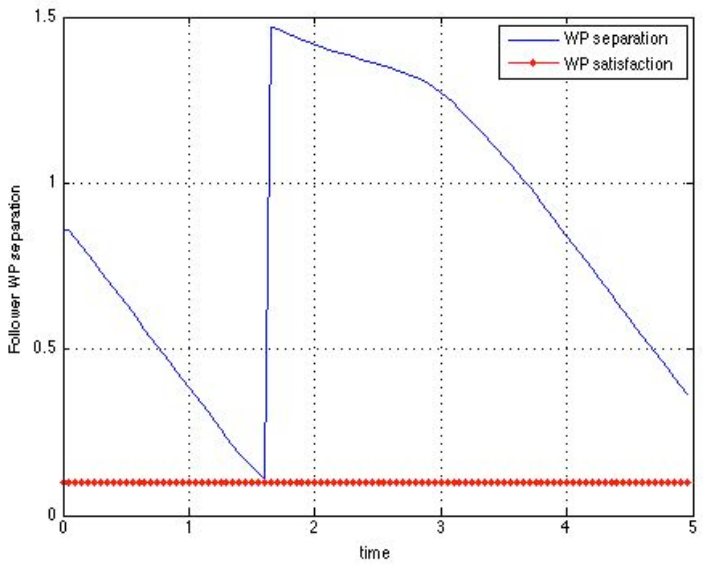

(b) Follower distance from waypoint.

Figure 6. Waypoint separation shown with satisfaction tolerance.

intersection. In the event that the conflict is not resolvable through flight path de-confliction, the UAVs use a collision avoidance scheme to immediately move away from each other. The control structure for the UAVs is hierarchical for more convenient implementation.

\section{Future work}

The authors will be improving the implementation of this patrolling strategy on a testbed of miniature helicopters flying in close proximity of one another. Operating three or more UAVs in the same confined space will require an algorithm with more predictive power. ${ }^{15}$ Future goals for this work include guiding three or more UAVs in real time in the same region. The authors will also be investigating algorithms that minimize the need for emergency collision avoidance.

\section{Acknowledgements}

This work was supported in part by the United States Air Force under grant number FA 8650-07-23744. The research leading to this work was also partly funded by Financiamento pluri-anual da Unidade de I\&D da FEUP ISR Porto by the FCT (Foundation for Science and Technology) under Phd grant $\mathrm{SFRH} / \mathrm{BD} / 40764 / 2007$.

\section{References}

\footnotetext{
${ }^{1}$ Girard, A. R., Howell, A. S., and Hedrick, J. K., "Border Patrol and Surveillance Missions using Multiple Unmanned Air Vehicles," IEEE Conference on Decision and Control, Atlantis, Paradise Island, Bahamas, December 2004, pp. 620-625.

2 "Unmanned Systems Roadmap," Tech. rep., Office of the Secretary of Defense, 2007.

${ }^{3}$ Bicchi, A. and Pallottino, L., "Conflict avoidance in networked mobility systems: towards decentralization," IEEE International Conference on Robotics and Automation, IEEE Robotics and Automation Society, Orlando, Florida, USA, 15-19 May 2006.

${ }^{4}$ Weitz, L. A., Hurtado, J. E., and Sinclair, A. J., "Decentralized Cooperative-Control Design for Multiple Formations," Journal of Guidance, Control, and Dynamics, Vol. 31, July-August 2008, pp. 970-979.

${ }^{5}$ Williams, E., "Airborne collision avoidance system," SCS '04: Proceedings of the 9th Australian workshop on Safety critical systems and software, Australian Computer Society, Inc., Darlinghurst, Australia, Australia, 2004, pp. 97-110.

${ }^{6}$ Mao, Z., Feron, E., and Bilimoria, K., "Stability and Performance of Intersecting Aircraft Flows Under Decentralized Conflict Avoidance Rules," IEEE Transactions on Intelligent Transportation Systems, Vol. 2, June 2001, pp. 101-109.

${ }^{7}$ Stipanovic, D., Inalhan, G., Teo, R., and Tomlin, C. J., "Decentralized overlapping control of a formation of unmanned aerial vehicles," Vol. 40, No. 8, 2004, pp. 1285-1296.

${ }^{8}$ Richards, A., Bellingham, J., Tillerson, M., and How, J. P., "Coordination and control of multiple uavs," in AIAA Guidance, Navigation, \& Control Conference, Monterey, CA, 2002.

${ }^{9}$ Dunbar, W. B. and Murray, R. M., "Model predictive control of coordinated multi-vehicle formation," 41th IEEE Conf.
} 
on Decision and Control, 2002.

${ }^{10}$ Olfati-Saber, R., Dunbar, W. B., and Murray, R. M., "Cooperative control of multi-vehicle systems using cost graphs and optimization," American Control Conference, December 14-17 2003.

${ }^{11}$ Giulietti, L. P. L. F. and Innocenti, M., "Autonomous formation flight," Vol. 20, No. 6, 2000 , pp. 34-44.

${ }^{12}$ Jardin, M., "Ideal free flight through multiple aircraft neighboring optimal control," American Control Conference, 2000. Proceedings of the 2000, Vol. 4, 2000, pp. 2879-2885 vol.4.

${ }^{13}$ Borrelli, F., Keviczky, T., and Balas, G. J., "Collision-free UAV Formation Flight Using Decentralized Optimization and Invariant Sets," 43rd IEEE Conference on Decision and Control, Paradise Island, Bahamas, December 14-17 2004.

${ }^{14}$ Richards, A. and How, J. P., "Aircraft Trajectory Planning With Collision Avoidance Using Mixed Integer Linear Programming," Proceedings of the American Control Conference, Anchorage, AK, May 2002, pp. 1936-1941.

${ }^{15}$ Chang, D. E., Shadden, S. E., Marsden, J. E., and Olfati-Saber, R., "Collision avoidance for multiple agent systems." IEEE Conference on Decision and Control., Maui, Hawaii, USA, December 2003, pp. 539-543.

${ }^{16}$ Tomlin, C., Pappas, G. J., and Sastry, S., "Conflict Resolution for Air Traffic Management: A Study in Multiagent Hybrid Systems," Vol. 43, No. 4, April 1998, pp. 509-521.

${ }^{17}$ Hoffmann, G. and Tomlin, C., "Decentralized cooperative collision avoidance for acceleration constrained vehicles," Decision and Control, 2008. CDC 2008. 47th IEEE Conference on, Dec. 2008, pp. 4357-4363.

${ }^{18}$ Hoffmann, G. M., Huang, H., Waslander, S. L., and Tomlin, C. J., "Quadrotor Helicopter Flight Dynamics and Control: Theory and Experiment," AIAA Guidance, Navigation, E Control Conference, Monterey, CA, Hilton Head, SC, August 2007.

19 de Sousa, J. B. and Girard, A. R., "Dynamic teams of networked vehicle systems," Portuguese Conference on Artificial intelligence, Covilha, Portugal, April 2007, pp. 230-235.

${ }^{20}$ Pallottino, L., Bicchi, A., and Frazzoli, E., "Probabilistic verification of decentralized multi-agent control strategies: a Case Study in Conflict Avoidance," American Control Conference, 200\%. ACC '07, July 2007, pp. 170-175. 